Apidologie, 1977, 8 (2), 111-127.

\title{
ACTION DE QUELQUES INSECTICIDES SUR UNE ABEILLE SOLITAIRE : MEGACHILE PACIFICA PANZ. (HYMENOPTERA, MEGACHILIDAE)
}

\section{Die Wirkung einiger Insektizide auf eine solitäre Biene : Megachile pacifica Panz. (Hymenoptera, Megachilidae)}

\author{
J.-N. TASEI, J. CAPOU et D. MICHAUD \\ Laboratoire de Zoologie, I.N.R.A. \\ 86600 Lusignan
}

\begin{abstract}
SUMMARY
effect of some Pesticides on a solitary bee (Megachile pacifica Panz). (Hymenoptera, Megachilidae)

This paper is concerned with the results of tests carried out in controlled or semi-controlled conditions on males of the leafcutting bee Megachile pacifica with commercial insecticides : Dialifor, Endosulfan, Phosalone, Trichlorfon, Oxydemeton-methyl and Pirimicarb.

Endosulfan and Dialifor are the most toxic whether by residues or by spray. Trichlorfon is hazardous in special conditions of use. Phosalone is moderately toxic when food is provided to the bees. Oxydemeton-methyl is little toxic and Pirimicarb not toxic at all.
\end{abstract}

\section{RÉSUMÉ}

L'article présente les résultats de tests insecticides exécutés en conditions contrôlées ou semi-contrôlées sur des mâles de l'abeille solitaire Megachile pacifica avec des produits commerciaux à base de Dialifor, Endosulfan, Phosalone, Trichlorfon, Oxydemeton-methyl et Pirimicarb.

L'Endosulfan et le Dialifor sont les plus toxiques, soit par les résidus, soit par pulvérisation. Le Trichlorfon est dangereux dans certaines conditions d'emploi. La Phosalone est moyennement toxique lorsque les insectes peuvent s'alimenter. L'Oxydemeton-methyl est faiblement toxique. Le Primicarb est inoffensif. 


\section{INT ROD UCTION}

Dans l'article intitulé ‘ Méthode de test de toxicité des pesticides applicable aux abeilles solitaires et plus particulièrement à Megachile pacifica » (TASEI, 1977), on a décrit en détail la méthode recommandée pour tester des pesticides sur Megachile pacifica. Rappelons brièvement les techniques : Deux types de tests sont proposés :

1) On contrôle la mortalité des insectes mâles qui sont en contact avec un papier-filtre chargé de résidus insecticides, placé dans une petite boîte en polystyrène à température constante sous un éclairage ininterrompu et sans aliments.

2) Un contrôle identique est effectué sur des insectes ayant subi une pulvérisation $d$ 'insecticides.

La mise au point de cette méthode a demandé certaines manipulations préliminaires qui permettent de mesurer l'incidence des choix techniques, tels que l'emploi d'individus de sexe mâle, la conservation des insectes après émergence avant le test, la diète imposée aux abeilles pendant le test. L'exposé des résultats de ces tests paraît nécessaire avant d'aborder la discussion générale. En second lieu sont présentés des tests spécialement effectués pour connaître l'action de quelques produits sur les mégachiles.

TABL. 1. - Concentration maximum des pulvérisations ou bains de chacun des 6 produits commerciaux testés sur Megachile pacifica.

TAB. 1. - Höchstkonzentration der Sprühmittel oder der Bäder jedes der sechs handelsüblichen Mittel, die an Megachile pacifica ïberprüft wurden.

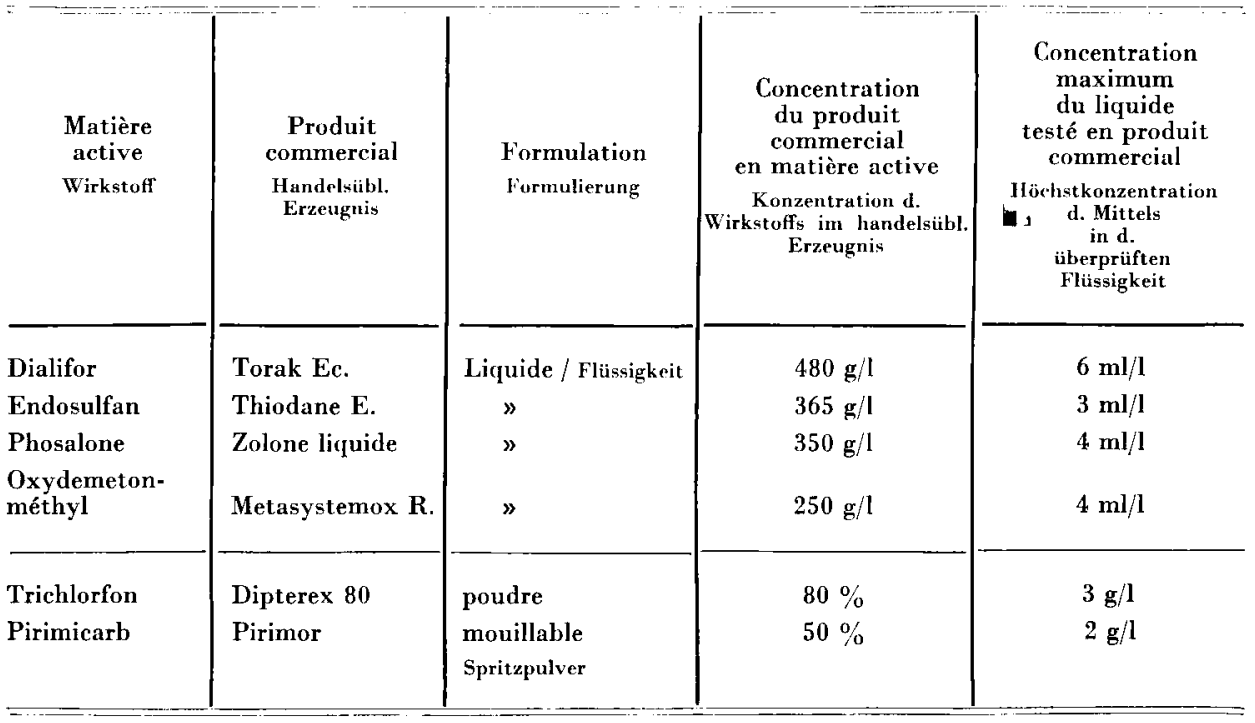


Les matières actives sont : la Phosalone, l'Endosulfan, le Dialifor, le Pirimicarb, le Trichlorfon, l'Oxydemeton-methyl (Tabl. 1). Les critères qui justifient le choix de ces substances sont : l'utilisation actuelle sur la luzerne pendant la phase fleurie de la Phosalone et de l'Endosulfan en France, du Trichlorfon et de l'Oxydemeton-methyl aux États-Unis et l'innocuité vis-à-vis de l'abeille domestique du Dialifor et du Pirimicarb (Cairaschi, communication personnelle) qui pourraient avoir une action sur certains ravageurs.

\section{TESTS PRELIMINAIRES}

\section{1. - Sur la différence de sensibilité des sexes}

Lorsqu'on dispose des mâles et des femelles de mégachiles sur des papiers à filtrer recouverts de résidus insecticides les taux définitifs de mortalité sont plus rapidement atteints chez les mâles que chez les femelles. Dans les lots non traités les femelles résistent moins bien que les mâles à la claustration (Tabl. 2).

TABL. 2. - Différence de sensibilité des mâles et des femelles de Megachile pacifica à l'égard des résidus de 4 insecticides.

TAB. 2. - Unterschiedliche Empfindlichkeit von Männchen und Weibchen (Megachile paeifica) hinsichtlich der Rückstände von vier Insektiziden.

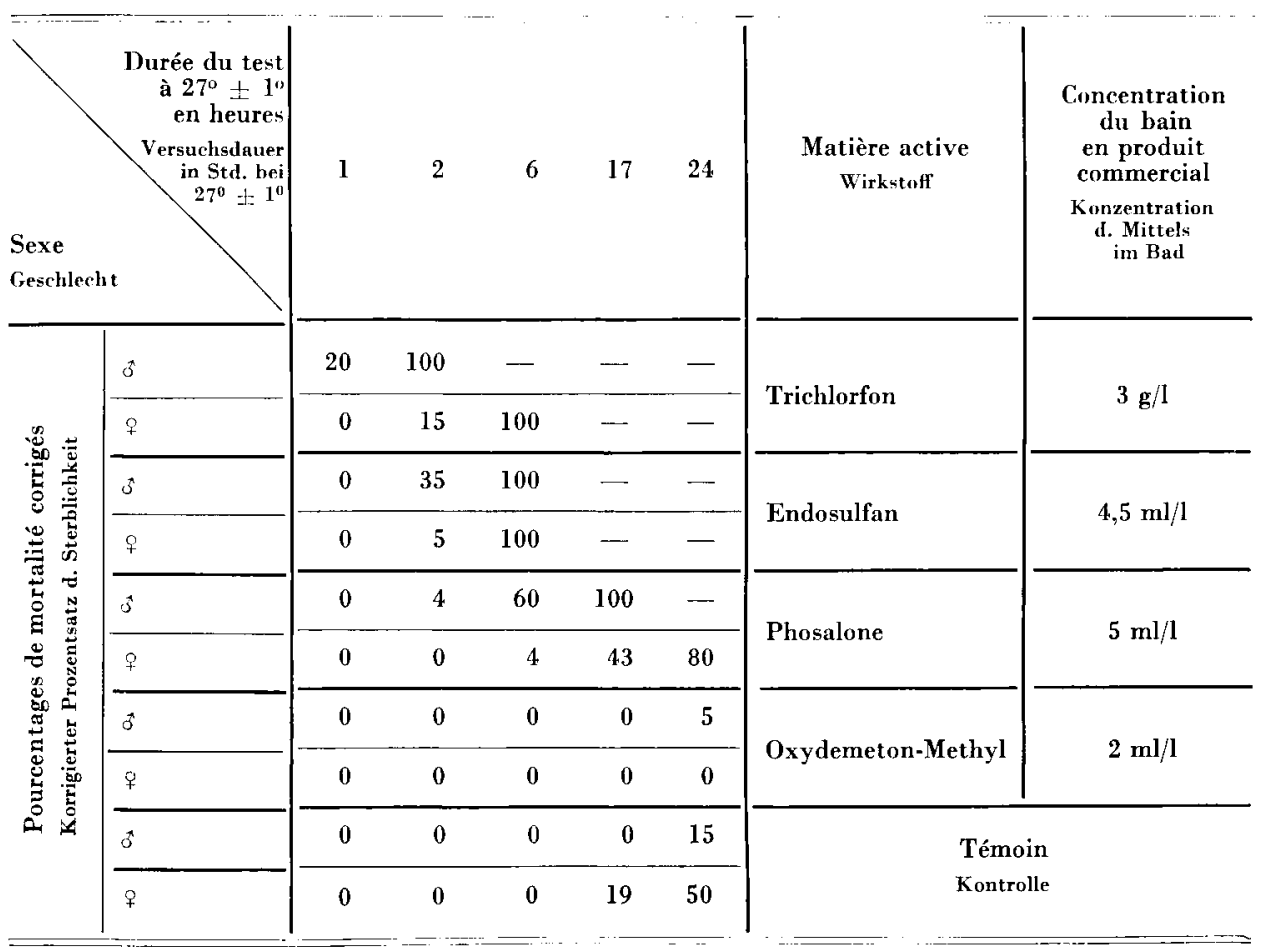




\section{2. - Sur l'effet de la conservation des insectes destinés aux tests, après leur émergence}

Lorsque la conservation à l'obscurité et à $18{ }^{\circ} \mathrm{C}$ dure 48 heures, la mortalité chez les mâles est nettement plus élevée que lorsqu'elle ne dure que 24 heures. Les lots non traités ne présentent pas de différence. Quant au classement des produits par ordre de toxicité croissante il ne varie pas (Tabl. 3). Il faut noter cependant que la différence des mortalités causées par l'Endosulfan et la Phosalone dans le c lot 48 heures » n'est pas significative ${ }^{1}$ alors que la légère mortalité causée par le Trichlorfon dans ce même lot est significative.

'TABL. 3. - Effet de la durée de conservation des mâles de Megachile pacifica laissês sans nourriture, sur leur sensibilité à 6 insecticides pulvérisés.

TAB. 3. - Einfluss der Aufbewahrungsdauer der nicht gefütterten Männchen von Megachile pacifica auf ihre Empfindlichkeit sechs gesprühten Insektiziden gegenüber.

\begin{tabular}{|c|c|c|c|c|}
\hline \multirow[t]{2}{*}{$\begin{array}{c}\text { Matière active } \\
\text { Wirkst off }\end{array}$} & \multicolumn{2}{|c|}{$\begin{array}{c}\text { Pourcentage de mortalité } \\
\text { au bout de } 20 \text { heures } \\
\text { à } 27^{\circ} \text { L } 1{ }^{\circ} \mathrm{C} \\
\text { Prozentsatz d. Sterblichkeit } \\
\text { nach } 20 \text { Std. bei } 27^{\circ} \pm 1^{\circ} \mathrm{C}\end{array}$} & \multirow{2}{*}{$\begin{array}{l}\text { Concentration } \\
\text { de la pulvérisation } \\
\text { en produit } \\
\text { commercial } \\
\text { Konzentration } \\
\text { d. Mittcls in d. } \\
\text { Sprühfliswigkeit }\end{array}$} & \multirow{2}{*}{$\begin{array}{c}\text { Température } \\
\text { de l'air } \\
\text { au moment } \\
\text { de la pulvérisation } \\
\text { Luftemperatur } \\
\text { beim Sprühen }\end{array}$} \\
\hline & Lot $24 \mathrm{~h}^{1}$ & Lot $48 \mathrm{~h}$ & & \\
\hline $\begin{array}{l}\text { Dialifor } \\
\text { Endosulfan } \\
\text { Phosalone } \\
\text { Oxydemeton-méthyl } \\
\text { Trichlorfon } \\
\text { Primicarb } \\
\text { Témoin } \\
\text { Kontrolle }\end{array}$ & $\begin{array}{r}73 \\
44 \\
32 \\
8 \\
0 \\
0 \\
0\end{array}$ & $\begin{array}{r}97 \\
84 \\
75 \\
30 \\
7 \\
0 \\
0\end{array}$ & 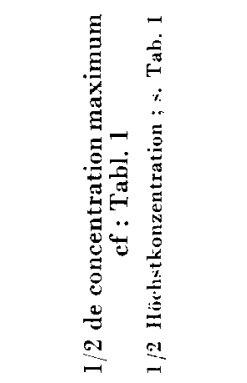 & $21-2{ }^{\circ} \mathrm{C}$. \\
\hline
\end{tabular}

1. Durée de la conservation à $18^{\circ} \mathrm{C}$ entre l'émergence des cocons et la pulvérisation.

1. Aufbewahrungsdauer bei $18{ }^{\circ} \mathrm{C}$ zwischen Schliipfen und Bespriihen.

\section{3. - Sur l'effet de la diète après un traitement par pulvérisation}

Après pulvérisation les abeilles sont divisées en 2 lots, l'un est traité selon la méthode recommandée sans nourriture en enceinte éclairée constamment et chauffée à $27 \pm 1{ }^{\circ} \mathrm{C}$, l'autre est mis dans une cage sous serre vitrée, où la tem-

1. Par le test de Kruskal et Waidis (non paramétrique). 
pérature maximum atteint $30^{\circ} \mathrm{C}$. La cage n'est pas éclairée la nuit et contient des pieds de Melilotus alba. Dans le cas des insecticides à action rapide (Dialifor et Endosulfan) le taux de mortalité est inférieur en cage. Dans tous les cas, exception faite de l'Oxydemeton-methyl, la mortalité au bout de 24 heures est nettement inférieure en cage. Les différences entre les deux modes de conservation après traitement sont surtout sensibles dans le cas du Dialifor et de la Phosalone (tabl. 4). Passé le délai de 24 heures les mortalités n'évoluent plus dans aucun des cas. Le classement des produits selon la toxicité est sensiblement modifié par la conservation en serre fleurie puisque l'Oxydemeton-methyl est légèrement plus toxique que la Phosalone et l'Endosulfan plus toxique que le Dialifor.

\section{MODE D'ACTION DES PRODUITS}

\section{1. - Évolution de la mortalité en fonction du temps et du mode d'application de l'insecticide}

Les figures 1 et 2 permettent de comparer la rapidité d'action et le degré de toxicité des insecticides selon le mode d'application (pulvérisation ou contact tarsal sur les résidus supportés par du papier-filtre). Le Dialifor et l'Endosulfan ont dans les deux cas une action rapide et très toxique. La Phosalone a dans les deux cas une action plus lente. Sa toxicité au bout de 20 heures est légèrement inférieure à celle du Dialifor dans le cas d'une pulvérisation. L'Oxydemeton-methyl est légèrement toxique uniquement après pulvérisation. Le Trichlorfon est très toxique par ses résidus avec une action retardée par rapport au Dialifor et à l'Endosulfan mais plus rapide que celle de la Phosalone. Il est inoffensif après pulvérisation (mortalité légère : 7 pour cent, significative par rapport au témoin). Le Pirimicarb n'est dangereux dans aucun des cas. Les mortalités enregistrées avec ce produit ne sont jamais significatives.

\section{2. - Influence de la température de l'air au moment de la pulvérisation}

A température basse $\left(9,5^{\circ} \mathrm{C}\right)$ le seul insecticide qui soit plus actif qu'à température moyenne $\left(21,5^{\circ} \mathrm{C}\right)$ est $\mathrm{l}^{\prime}$ Oxydemeton-methyl. Une augmentation de toxicité évidente s'observe à $21,5^{\circ} \mathrm{C}$ avec le Dialifor, l'Endosulfan et la Phosalone (tabl. 5). Il faut noter qu'à 9,5 ${ }^{\circ} \mathrm{C}$ les différences de mortalité entre Endosulfan, Phosalone et Oxydemeton-methyl ne sont pas significativement différentes. A $21,5^{\circ} \mathrm{C}$ la seule différence non significative est celle qui est observée entre les mortalités dues à l'Endosulfan et à la Phosalone. Le Trichlorfon est inoffensif. Une légère toxicité apparaît à $21,5^{\circ} \mathrm{C}$. Lors de deux tests qui ne sont pas exposés ici on a effectué avec Trichlorfon à $3 \mathrm{~g} / \mathrm{l}$ des pulvérisations à $22^{\circ} \mathrm{C}$ et à 
TABL. 4. - Influence de la prise de nourriture sur la sensibilité des mâles de Megachile pacifica aux pulvérisations d'insecticides.

TAB. 4. - Einfluss der Nahrungsaufnahme auf die Empfindlichkeit der Megachile-Männchen dem Besprühen mit Insektiziden gegenüber.

\begin{tabular}{|c|c|c|c|c|c|}
\hline \multirow{2}{*}{$\begin{array}{c}\text { Matière active } \\
\text { Wirkstoff }\end{array}$} & \multirow{2}{*}{$\begin{array}{c}\text { Durée } \\
\text { du test } \\
\text { en heures } \\
\text { Vermuchidauer } \\
\text { in Sitd. }\end{array}$} & \multicolumn{2}{|c|}{$\begin{array}{c}\text { Pourcentage de mortalité } \\
\vdots ; \text { Sterblichkeit }\end{array}$} & \multirow{2}{*}{$\begin{array}{l}\text { Concentration } \\
\text { de Ia pulvérisation } \\
\text { en produit } \\
\text { commercial } \\
\text { Konzentration d. } \\
\text { Mittels in d. } \\
\text { Sprïhlösung }\end{array}$} & \multirow{2}{*}{$\begin{array}{l}\text { Température } \\
\text { de l'air au moment } \\
\text { de la pulvérisation } \\
\text { Lufttemperatur } \\
\text { wäbrend d. Sprïhens }\end{array}$} \\
\hline & & $\mid \begin{array}{ccc}27^{0} & I^{1} \\
\text { à jeun } & \\
\text { bei } 27^{0} & 1 \\
\text { ungefüttert }\end{array}$ & $\begin{array}{c}\text { en serre }{ }^{2} \\
\text { avec fleurs } \\
\text { in Treibhaus, } \\
\text { mit Blaten }{ }^{2}\end{array}$ & & \\
\hline Dialifor & $\begin{array}{r}4 \\
16 \\
24\end{array}$ & $\begin{array}{l}64 \\
97 \\
98\end{array}$ & $\begin{array}{l}50 \\
50 \\
50\end{array}$ & \multirow{7}{*}{ 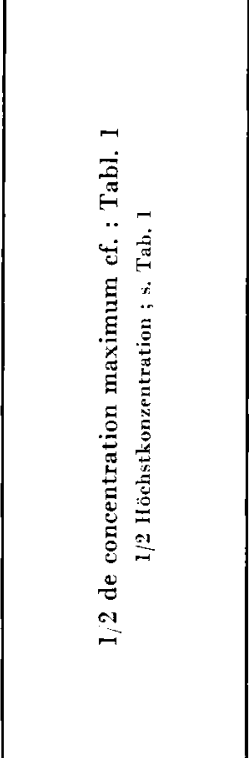 } & \multirow{7}{*}{$21,5 \cap \mathrm{C}$} \\
\hline Endosulfan & $\begin{array}{r}4 \\
16 \\
24\end{array}$ & $\begin{array}{l}70 \\
82 \\
85\end{array}$ & $\begin{array}{l}58 \\
58 \\
63\end{array}$ & & \\
\hline Phosalone & $\begin{array}{r}4 \\
16 \\
24\end{array}$ & $\begin{array}{r}3 \\
70 \\
79\end{array}$ & $\begin{array}{l}11 \\
24 \\
25\end{array}$ & & \\
\hline Oxydemeton-méthyl & $\begin{array}{r}4 \\
16 \\
24\end{array}$ & $\begin{array}{r}2 \\
22 \\
35\end{array}$ & $\begin{array}{r}8 \\
15 \\
32\end{array}$ & & \\
\hline Trichlorfon & $\begin{array}{r}4 \\
16 \\
24\end{array}$ & $\begin{array}{r}1 \\
5 \\
13\end{array}$ & $\begin{array}{l}1 \\
1 \\
1\end{array}$ & & \\
\hline Pirimicarb & $\begin{array}{r}4 \\
16 \\
24\end{array}$ & $\begin{array}{l}0 \\
0 \\
0\end{array}$ & $\begin{array}{l}0 \\
0 \\
0\end{array}$ & & \\
\hline $\begin{array}{l}\text { Témoin } \\
\text { Kontrolle }\end{array}$ & $\begin{array}{r}4 \\
16 \\
24\end{array}$ & $\begin{array}{l}0 \\
0 \\
0\end{array}$ & $\begin{array}{l}0 \\
0 \\
0\end{array}$ & & \\
\hline
\end{tabular}

1. L'éclairage est constant pendant le test.

2. Entre la $4^{\mathrm{c}}$ et la $16^{\mathrm{e}}$ heure du test il y a eu l'arrêt d'activité nocturne. Ia température maximum a été $30^{\circ} \mathrm{C}$. Les fleurs sont du Melilotus alba.

1. Dauerbeleuchtung während des Versuchs.

2. Während der 4. u. 16. Stunde trat eine Unterbrechung der nächılichen Tätigkeit ein. Die Höchsttemperatur betrug $30^{\circ} \mathrm{C}$. Die Blüten sind Melilotus alba. 


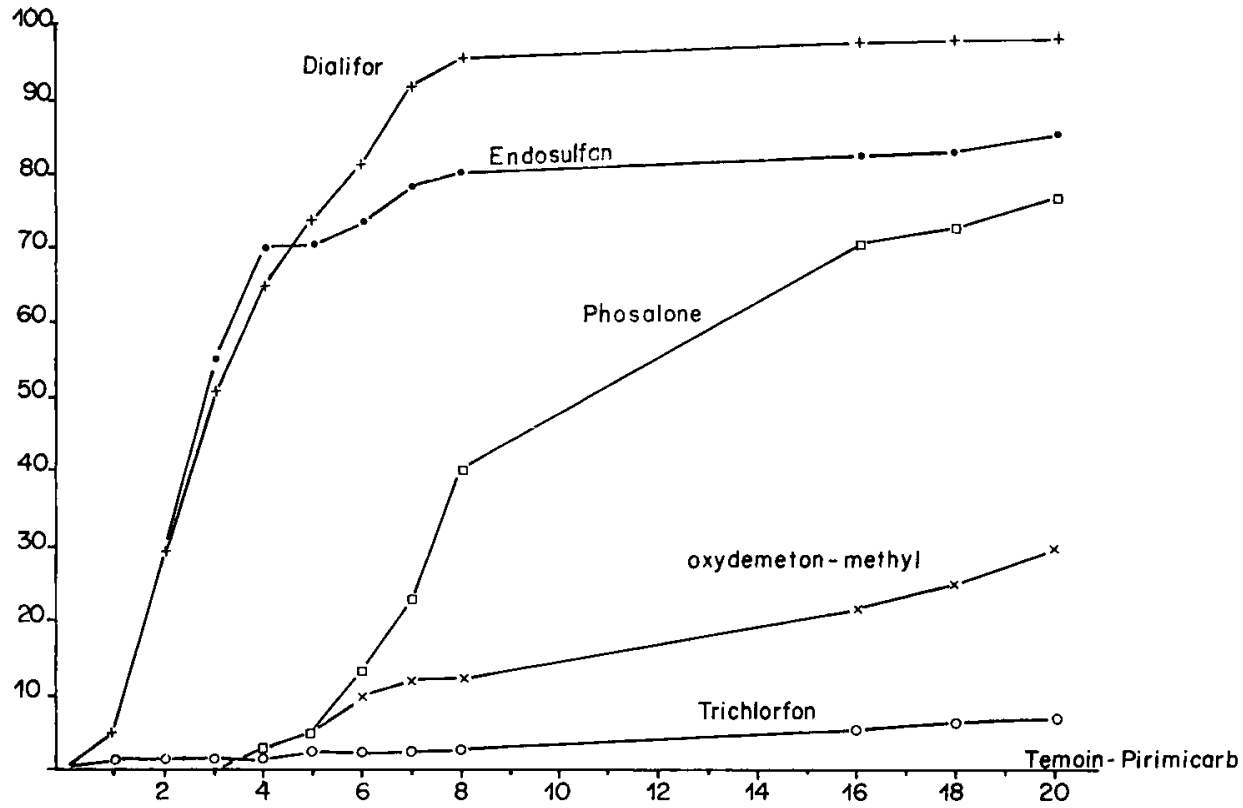

FIg. I. - Mortalitê des mâles de Megachile pacifica après pulvêrisation de 6 insecticides effectuée en plein air à $21,5^{\circ} \mathrm{C}$.

Horizontalement : durée de la conservation à $27^{\circ} \pm 1^{\circ} \mathrm{C}$ en heures. Verticalement : Pourcentage de mortalité.

Aв8. 1. - Sterblichkeit der Männchen von Megachile pacifica nach Besprühen mit sechs Insektiziden im Freien bei $21,5{ }^{\circ} \mathrm{C}$.

Abszisse $=$ Haltungsdauer bei $27^{\circ} \pm 1^{\circ} \mathrm{C}$ in Stunden. Ordinate $=$ Sterblichkeitsquote in \%. 


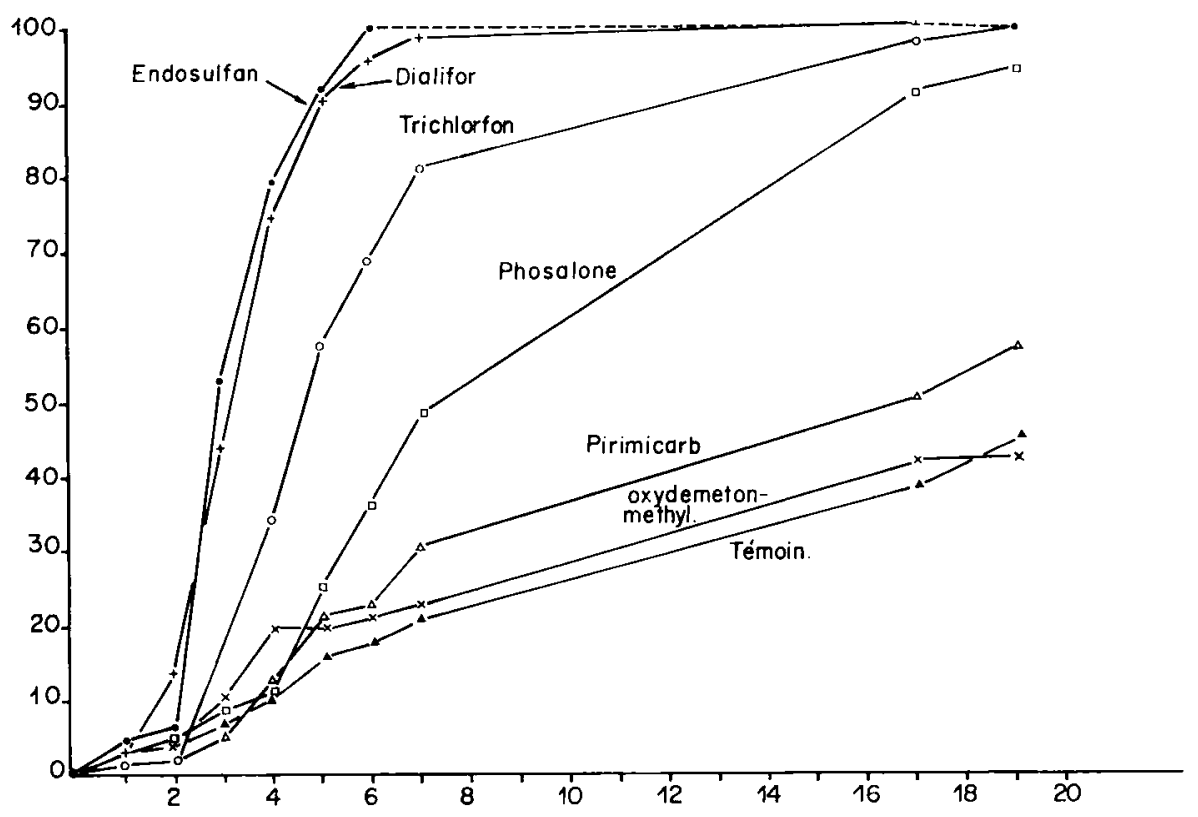

Fic. 2. - Mortalité des mâles Megachile pacifica en contact avec les résidus de 6 insecticides sur papier-filtre.

Horizontalement : Durée de la conservation des insectes à $27^{\circ}+1^{\circ} \mathrm{C}$. Verticalement : Pourcentage de mortalité.

Aвв. 2. - Sterblichkeit der Männchen von Megachile pacifica, die mit den Rückständen von sechs Insektiziden auf Fillerpapier in Berührung kamen.

Abszisse $=$ Dauer der Insektenhaltung bei $27^{\circ} \perp 1^{\circ} \mathrm{C}$. Ordinate $=$ Sterblichkeitsquote in $\%$. 
$24{ }^{\circ} \mathrm{C}$. Au bout de 20 heures la mortalité dans le premier cas ne dépasse pas $20 \%$, dans le second elle atteint $95 \%$. Si l'on emploie la dose de $1 \mathrm{~g} / \mathrm{l}$ la mortalité est infime, même à la température de $26^{\circ} \mathrm{C}$.

TABL. 5. - Effet de la température de l'air au moment de la pulvêrisation sur la sensibilité des mâles de Megachile pacifica à 6 insecticides.

ТАВ. 5. - Einfluss der Lufttemperatur während des Sprühens auf die Empfindlichkeit der Männchen von Megachile pacifica bei sechs Insektiziden.

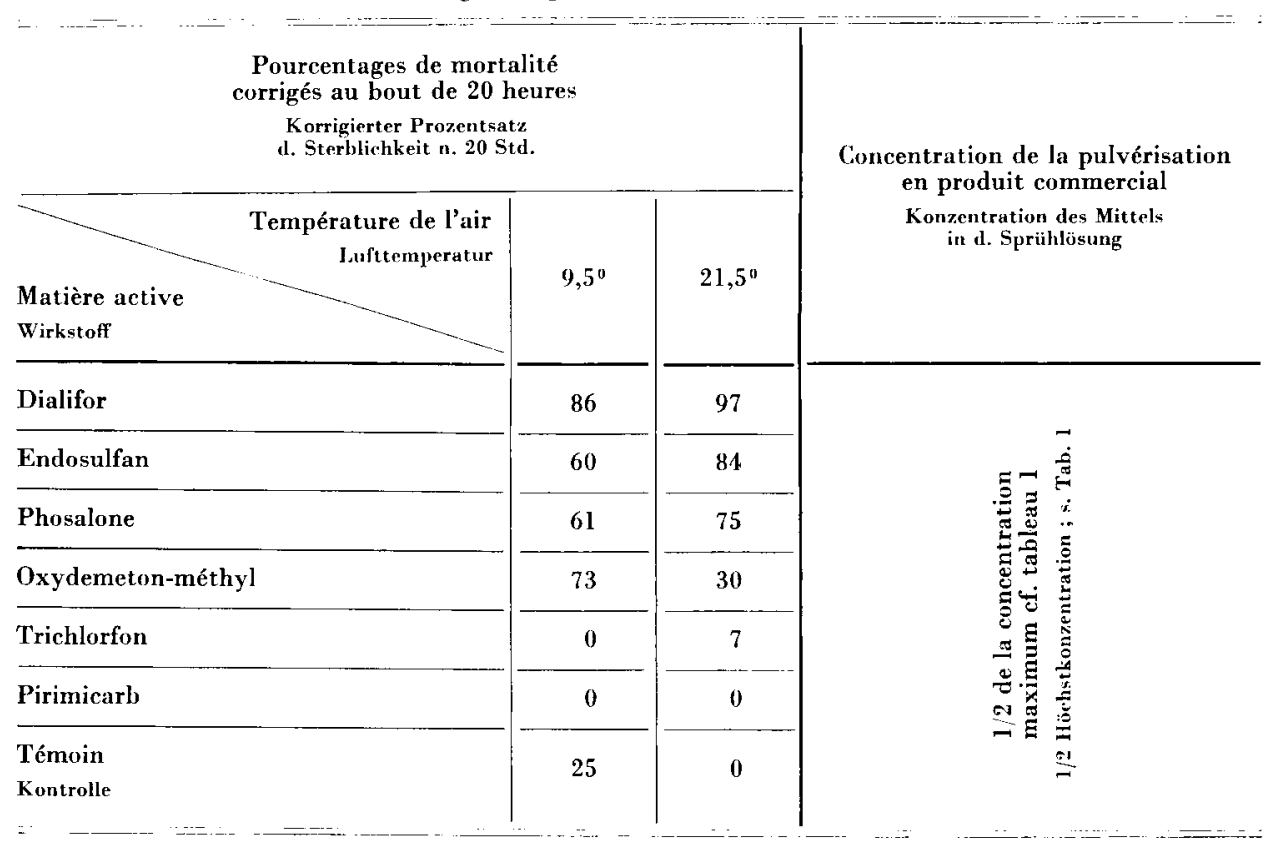

\section{3. - Influence de la dose}

Lorsque l'on diminue la concentration du liquide pulvérisé de moitié, les mortalités observées sont en régression très nette pour l'Endosulfan, assez nette pour l'Oxydemeton-methyl et le Trichlorfon, tandis que la Phosalone conserve presque la même action (tabl. 6).

\section{4. - Persistance de la toxicité des résidus}

Lorsqu'on laisse vieillir les résidus d'insecticides de 1 heure à 5 jours, leur toxicité commence à décroître plus ou moins sensiblement dès le second jour exception faite du Dialifor et de l'Endosulfan qui restent presque aussi actifs au cinquième jour qu'au premier. La diminution de toxicité la plus évidente est cclle du Trichlorfon qui au cinquième jour tue 2 fois moins d'insectes qu'au premier. La Phosalone est un cas intermédiaire puisqu'au cinquième jour la toxicité se maintient à un niveau assez élevé (tabl. 7). 
TABL. 6. - Effet de la concentration de la pulvérisation sur la mortalité des mâles de Megachile pacifica.

Тав. 6. - Einfluss der Konzentration des Sprühmiltels auf die Sterblichkeit der Megachile-Männchen.

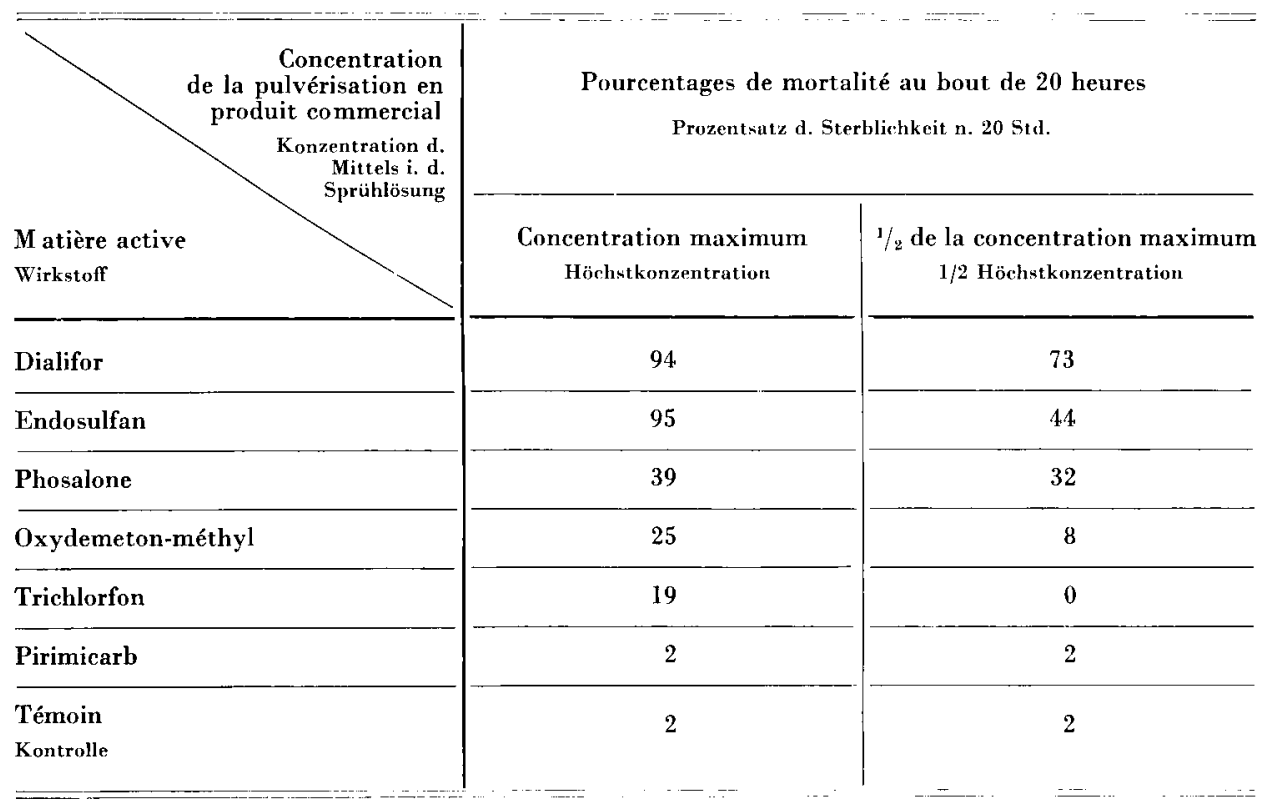

TaBL. 7. - Persistance de la toxicité de 6 insecticides à l'égard des mâles de Megachile pacifica.

TAB. 7. - Dauer der Giftwirkung von sechs Insektiziden in bezug auf die Männchen von Megachile pacifica.

\begin{tabular}{|c|c|c|c|c|c|}
\hline \multirow{2}{*}{$\begin{array}{l}\text { Matière } \\
\text { active } \\
\text { Wirkstoff }\end{array}$} & \multicolumn{3}{|c|}{$\begin{array}{l}\text { Pourcentages de mortalité } \\
\text { corrigés au bout de } 20 \text { heures } \\
\text { Korrigierter Prozentsatz } \\
\text { d. Sterblichkeit n. } 20 \text { Std. }\end{array}$} & \multirow{2}{*}{$\begin{array}{c}\text { Année d'obtention } \\
\text { des résultats } \\
\text { Jahr der crhultenen } \\
\text { Ergebnisse }\end{array}$} & \multirow{2}{*}{$\begin{array}{l}\text { Concentration } \\
\text { du liquide utilisé } \\
\text { en produit } \\
\text { commercial } \\
\text { Konzentration d. } \\
\text { Mittels in d. benutzten } \\
\text { Flüsigkeit }\end{array}$} \\
\hline & $\begin{array}{l}1 \text { heure } \\
\text { 1 Stunde }\end{array}$ & $\begin{array}{l}2 \text { jours } \\
2 \text { Tage }\end{array}$ & $\begin{array}{c}5 \text { jours } \\
5 \text { Tage }\end{array}$ & & \\
\hline Dialifor & 100 & 100 & 90 & 1975 & $6 \mathrm{ml} / \mathrm{l}$ \\
\hline Endosulfan & 100 & 100 & 96 & 1974 & $2,5 \mathrm{ml} / 1$ \\
\hline Phosalone & $\begin{array}{r}100 \\
96\end{array}$ & $\begin{array}{l}76 \\
60\end{array}$ & $\begin{array}{l}73 \\
73\end{array}$ & $\begin{array}{l}1974 \\
1975\end{array}$ & $\begin{array}{c}2,5 \mathrm{ml} / 1 \\
4 \mathrm{ml} / \mathrm{l}\end{array}$ \\
\hline Trichlorfon & 100 & 83 & 45 & 1974 & $1,5 \mathrm{~g} / 1$ \\
\hline Oxydemeton-méthyl & 0 & 0 & 0 & 1974 & $1 \mathrm{ml} / \mathrm{l}$ \\
\hline Pirimicarb & 0 & 0 & 0 & 1975 & $2 \mathrm{~g} / 1$ \\
\hline
\end{tabular}




\section{DISCUSSION}

\section{1. - Sur les techniques}

Le choix des insectes mâles pour tester l'action des insecticides s'avère plus intéressant que celui des femelles. En effet, ces dernières sont moins résistantes aux conditions de conservation en étuve que nous pratiquons. Par ailleurs, elles sont moins mobiles que les mâles et c'est probablement pour cette raison que leur réaction aux insecticides est moins rapide.

On a vu que la durée de conservation des insectes destinés aux tests influe beaucoup sur les taux de mortalité. Les individus émergés de leur cocon depuis 2 jours sont beaucoup plus sensibles aux traitements que ceux qui ont émergés depuis 24 heures. Cette différence de résistance ne peut être décelée par comparaison avec des lots témoins puisqu'ils ne présentent aucune différence de mortalité. En conséquence si l'on veut comparer les résultats de 2 tests il est important de choisir 2 lots d'insectes ayant eu des durées de conservation égales. Afin de ne pas faire apparaître de sensibilités trop éloignées des réactions de l'insecte dans son milieu naturel, il est recommandé de ne pas conserver les mégachiles émergés plus de 24 heures.

Il est plus facile de conserver les insectes, pendant ou après leur contact avec l'insecticide, sans nourriture. Cependant, il est certain que leur libération en serre fleurie est une bonne approche expérimentale des réactions de l'espèce lors de traitements effectués dans les conditions naturelles. Dans ces conditions semi-artificielles 4 produits sur 5 ont causé moins de pertes que lors de la conservation en étuve. La différence d'action varie suivant le produit, c'est ainsi qu'avec la Phosalone on observe la régression de mortalité la plus forte tandis que l'Oxydemeton-methyl conserve la même action.

\section{2. - Sur les résultats}

Les tests que nous avons présentés ne sont pas assez nombreux pour nous permettre de juger de l'effet de traitements appliqués en plein champ. En particulier les expériences sur papier-filtre obligent les insectes à rester en contact avec les résidus de façon constante et ne peuvent refléter les phénomènes observables dans les champs traités. Ces expériences de laboratoire permettent cependant de classer les produits selon leur toxicité et de ranger dans la catégorie ( produit dangereux ou suspect ) tout insecticide occasionnant au cours d'un des tests une mortalité non négligeable (supérieure à $30 \%$ par exemple). Le tableau no 8 donne une appréciation générale sur la toxicité des 6 insecticides étudiés. L'estimation tient compte non seulement des tests exposés ici, mais aussi d'autres expériences qui n'ont pas été présentées dans ces pages afin de ne pas alourdir l'ensemble. 
TABL. 8. - Appréciation globale de la toxicité des insecticides pour les mâles de Megachile pacifica.

TaB. 8. - Gesamtbewertung der Toxizität der Insoktizide für die Männchen von Megachile pacifica.

\begin{tabular}{|c|c|c|c|}
\hline $\begin{array}{c}\text { Conditions } \\
\text { de conservation } \\
\text { Aufbewahrungsbedigungen }\end{array}$ & \multicolumn{2}{|c|}{$\begin{array}{c}\text { En étuve } 27{ }^{\circ} \mathrm{C}+\text { lumière jour et nuit } \\
+ \text { nourriture nulle } \\
\text { Thermostat } 27{ }^{\circ} \mathrm{C} \text { Dauerbcleuehtung } \\
\text { ohne Nahrung }\end{array}$} & $\begin{array}{l}\text { En serre avec fleurs } \\
\text { Im Treibhaus +f. Bliiten }\end{array}$ \\
\hline $\begin{array}{l}\begin{array}{c}\text { Mode d'application } \\
\text { Me l'insecticide } \\
\text { Anwendungsart }\end{array} \\
\text { Wirkstoff }\end{array}$ & $\begin{array}{l}\text { Résidus âgés } \\
\text { de } 1 \text { à } 5 \text { jours } \\
\text { sur papier filtre } \\
\text { 1-5 Tage alte Rückständt } \\
\text { nuf Filterpapier }\end{array}$ & $\begin{array}{l}\text { Pulvérisation } \\
\text { en plein air } \\
\text { Besprïhen im Jireien }\end{array}$ & $\begin{array}{l}\text { Pulvérisation } \\
\text { en plein air } \\
\text { Besprühen in Freien }\end{array}$ \\
\hline Dialifor & T.T. & T.T. & $\mathrm{T}$. \\
\hline Endosulfan & T.T. & T. à T.T. & $\mathrm{T}$. \\
\hline Trichlorfon & T. à T.T. & $\begin{array}{l}\text { T.T. si } \theta \text { air }>23{ }^{\circ} \mathrm{C} \\
0-\text { si } \theta \text { air }<23{ }^{\circ} \mathrm{C} \\
\text { ou si concentra- } \\
\text { tion }<1,5 \mathrm{~g} / \mathrm{l} \\
\text { T.T. wenn } \theta \mathrm{Luft} \\
23^{\circ} \mathrm{C} \\
\text { Owenn } \theta \text { Luft } \\
\text { oder wenn Konzen- } \\
\text { tration }<1.5 \mathrm{~g} / 1\end{array}$ & $\begin{array}{c}0 \text { si } 0 \text { air }<23{ }^{\circ} \mathrm{C} \\
\text { Owenn } \theta \text { Luft }<23 \quad{ }^{\circ} \mathrm{C}\end{array}$ \\
\hline Phosalone & T. à T.T. & $\mathrm{T}$. & $\iota$. \\
\hline Oxydemeton-méthyl & 0 & $\begin{aligned} \text { t. à } \theta \text { air }>16^{\circ} \mathrm{C} \\
\text { t hei } \theta \text { Luft }>16^{\circ} \mathrm{C}\end{aligned}$ & $t$. \\
\hline Pirimicarb & 0 & 0 & 0 \\
\hline
\end{tabular}

Très Toxique / Sehr giftig $=$ T.T. (75 à $100 \%$ de mortalité) $(75-100 \%$ Tote)

Toxique / Giftig = T. (35 ̀̀ $75 \% 》 ")(35-75 \%$ Tote)

peu Toxique / wenigg giftig $=\quad t$. $(10$ à $30 \% » \quad 》)(10.30 \%$ Tote $)$

non Toxique / nicht giftig = 0 ( 0 à $5 \% 》 \#)(0-5 \%$ Tote)

Le Pirimicarb est un produit parfaitement inoffensif quel que soit le mode d'application.

L'Oxydemeton-methyl a des résidus inoffensifs et la pulvérisation cause des pertes légères en moyenne.

La Phosalone est relativement dangereuse selon nos résultats de laboratoire. Cependant son application en fin de journée sur des champs où étaient installés des nichoirs à mégachiles, n'a provoqué aucune perte visible.

Le Trichlorfon a une action très variable selon son mode d'application et les conditions de température et de concentration : tantôt inoffensif, tantôt très toxique. Les résidus sont toujours assez dangereux quelle que soit la concentration. 
Le Dialifor et l'Endosulfan sont très toxiques quelles que soient les conditions d'emplois.

\section{3. - Sur les travaux étrangers}

Aux États-Unis de nombreuses publications font part de travaux concernant la protection des insectes pollinisateurs de la luzerne lors de traitements insecticides. Plusieurs auteurs se sont penchés sur la question et plusieurs méthodes ont été utilisées. Des tests en laboratoire ou en conditions semiartificielles (serre ou cage) ont permis d'évaluer la toxicité d'un grand nombre de produits à l'égard de Megachile pacifica, Nomia melanderi et de l'abeille domestique. Ces auteurs déconseillent formellement l'emploi de certains produits pendant la floraison de la luzerne, quel que soit le moment de la journée où l'on effectue la pulvérisation. Certaines matières actives sont en effet dangereuses pour l'une ou l'autre des espèces d'apoïdes citées, soit par les résidus, soit par le dépôt direct du produit sur le corps de l'insecte, c'est le cas entre autres de l'Endosulfan jugé dangereux pour les mégachiles. D'autres produits pourraient être utilisés en cours de floraison si l'on prend la précaution de les pulvériser en dehors des heures de vol des abeilles de tout genre. C'est le cas des insecticides dont le dépôt direct sur le corps des abeilles cause de grosses pertes, mais dont les résidus laissés sur le feuillage sont peu toxiques par exemple : l'Oxydemeton-methyl, la Phosalone, le Trichlorfon (Johansen 1969-1971).

Les auteurs américains ne disent rien de l'action du Dialifor et du Pirimicarb sur les mégachiles, ils donnent par contre de nombreuses informations sur l'action du Trichlorfon et de l'Oxydemeton-methyl. Ce dernier produit n'a pas d'action résiduelle (Johansen, 1969) et il ne contamine pas de façon dangereuse le nectar de luzerne quelle que soit la dose d'emploi (Mizuta et Johansen, 1972). Le Trichlorfon a été testé à plusieurs reprises, avec divers adjuvants. Les résidus des formulations les moins toxiques occasionnent 5 à $20 \%$ de pertes (Johansen et al. 1963, Johansen et Eves, 1967) par contre, la modification de l'acidité peut entraîner jusqu'à $60 \%$ de mortalité (JoHANSEN et Eves, 1972). Le Trichlorfon est tout de même jugé « non toxique " pour le mégachile, par référence aux essais effectués avec le produit non acidifié (JoHANsEn et EvEs, 1973). D'après Torchio (communication personnelle) les traitements pendant la floraison qui donnent satisfaction aux producteurs de semences de luzerne américains sont des pulvérisations à la tombée du jour, faites avec un mélange d'Oxydemeton-méthyl et de Trichlorfon.

Les travaux de laboratoire des chercheurs américains font ressortir les différences de sensibilité aux matières actives entre espèces. JoHANSEN et al. (1963), Torchio (1973) ont calculé les D.L. 50 d'un certain nombre d'insecticides chez $M$. pacifica et chez l'abeille domestique. Ils ont tous les deux constaté 
que chez les mégachiles les D.L. 50 étaient beaucoup plus variables que chez l'abeille domestique. Par ailleurs, les résultats de Torchio, plus précis que ceux de Johansen et al., montrent que les mégachiles sont nettement moins sensibles que l'abeille domestique à la plupart des produits, c'est le cas du Trichlorfon et de l'Oxydemeton-methyl (D.L. $50 \mathrm{du}$ mégachile 2 fois plus forte que celle de l'abeille domestique). Par contre, quelques produits sont plus toxiques pour le mégachile que pour l'abeille. (Malathion, Phosphamidon).

L'analyse de la bibliographie étrangère présente certaines difficultés. Ainsi les méthodes employées ne sont pas toujours précisées et de toute façon ne sont pas standardisées, ce qui peut expliquer des résultats contradictoires. Dans l'ensemble l'opinion des auteurs américains sur les produits que nous avons utilisés concorde avec nos résultats. La seule discordance concerne la toxicité des résidus du Trichlorfon à l'égard de $M$. pacifica, elle pourrait être due à la formulation du produit commercial, différente aux États-Unis.

\section{CONCLUSIONS}

1) L'espèce Megachile pacifica est une abeille solitaire bien adaptée aux tests insecticides en laboratoire. Les caractéristiques de sa biologie et de son élevage favorisent la standardisation de ces tests.

2) Ces tests en laboratoire présentent deux sortes d'intérêt : 1 - ils indiquent globalement le classement des insecticides selon leur toxicité d'une façon rapide et relativement économique : Les insecticides les plus dangereux sont l'Endosulfan et le Dialifor. Le Trichlorfon est dangereux dans certaines conditions d'emploi, la Phosalone est moyennement toxique, l'Oxydemetonmethyl est faiblement toxique et le Pirimicarb est inoffensif. 2 - Ils permettent de dissocier l'action des résidus de l'action de la pulvérisation : Les deux actions sont semblables chez l'Endosulfan, le Dialifor et la Phosalone. Chez le Trichlorfon seuls les résidus sont toujours toxiques, tandis que seule la pulvérisation d'Oxydemeton-méthyl peut provoquer quelques pertes.

3) Les techniques recommandées imposent aux insectes des conditions plus rudes (privation de nourriture ou contact permanent avec les résidus) que celles qui existent dans les champs traités. Pour cette raison une généralisation de nos résultats serait dangereuse, les tests en conditions semi-naturelles le prouvent (réduction très sensible de la mortalité due à la Phosalone quand les insectes peuvent se nourrir). Ces tests sont uniquement la première étape dans les recherches destinées à protéger les insectes pollinisateurs de la luzerne. Les prochaines études seront des tests en conditions naturelles. 
4) Nos résultats confirment une fois de plus que les produits déclarés (c non toxiques pour les abeilles ${ }^{1}$ ) sont en fait parfois très toxiques pour les espèces solitaires telles que $M$. pacifica (exemple : l'Endosulfan). L'inverse peut se rencontrer (exemple l'Oxydemeton-methyl). Il serait done souhaitable qu'en l'absence de tests appliqués aux abeilles sauvages, les informations destinées aux utlisateurs d'insecticides portent la mention moins ambigüe : ( non toxique pour l'Abeille domestique ) et que par ailleurs on étende systématiquement les mesures de protection à d'autres représentants de la faune pollinisatrice. Il en résulterait une sélection très sévère parmi les quelques insecticides reconnus non toxiques pour l'Abeille domestique. Pour protéger à la fois les cultures et les insectes pollinisateurs, techniciens et praticiens seraient peut-être amenés à choisir des produits moins spécifiques (le Pirimicarb est un exemple de produit très spécifique) mais dont les résidus sont peu rémanents (quelques heures) ou ne présentent pas de danger pour les insectes pollinisateurs. Leur application ne pourrait avoir lieu qu'en dehors des heures de vol des abeilles, de préférence le soir. Ce type d'insecticide est assez bien représenté par l'Oxydemeton-methyl.

\section{Reçu pour publication en février 1977.} Eingegangen im Februar 1977.

\section{REMERCIEMENTS}

Nous remercions les Sociétés Bayer-Phytochim, la Littorale, Procida, Rhône-Poulenc, Sopra, de nous avoir aimablement procuré les échantillons des insecticides testés.

\section{ZUSAMMENFASSUNG}

In einer vorangegangenen Arbeit (TASEI, 1977) wurde ein Verfahren beschrieben, das zur Überprüfung der Giftwirkung von Pflanzenschutzmitteln auf Megachile pacifica empfohlen wird. Die angewandten Verfahrensweisen sind : 1. Versuche auf Filterpapier, das mit Rückständen von Pflanzenschutzmitteln beschickt wurde. Die Untersuchungen wurden bei konstanten Temperaturen, Dauerbeleuchtung und ohne Fütterung durchgeführt. 2. Sterblichkeitsnachweis bei Insekten, die mit Insektiziden besprüht wurden.

Sechs handelsübliche Insektizide wurden überprüft. Ihre Wirkstoffe sind : Phosalon, Endosulfan, Dialifor, Pirimicarb, Trichlorfon, Methyl-Oxydemeton (Tab. 1).

\section{Ergebnisse der vorläufigen Versuche}

- Eine vergleichende Untersuchung ergab, dass die Reaktion der Männchen den Rückständen der Insektizide gegenüber rascher erfolgte als die der Weibchen. Ausserdem zeigte sich, dass die Weibchen das Gekäfigtsein weniger gut überstanden als die Männchen (Tab. 2).

1. Terme non spécifique conduisant à interpréter la formule dans un sens général et à utiliser dans la pratique les produits de façon dangereuse. 
Wenn Männchen 48 Stunden anstatt 24 Stunden lang aufbewahrt werden, bevor sie mit dem Insektizid in Berührung kommen, ist ihre Lmpfindlichkeit dem Mittel gegenüber grösser (Tab. 3).

- Nach dem Besprühen mit dem Insektizid hält man die Bienen entweder in Kunststoff (Polystyren)-Kästchen ohne Fütterung oder im Gewächshaus, in dem Kästen mit Melilotus alba stehen. Die Sterblichkeit im Gewächshaus ist bei allen Mitteln, mit Ausnahme von MethylOxydemeton, deutlich geringer (Tab. 4).

\section{Ergebnisse, die die Wirkungsweise der Pflanzenschutzmittel betreffen}

- Ein Vergleich von Schnelligkeit der Wirkung und Giftigkeit der Mittel je nach Anwendungsart (Besprühen oder Kontakt mit Rückständen) ergibt eine Klassifizierung der Insektizide (Abb. 1 u. 2). Dialifor und Endosulfan erwiesen sich in allen Fällen als rasch wirksam und sehr giftig. Phosalon wirkt langsam. Trichlorfon ist nur in seinen Rückständen giftig, im Gegensatz zu Methyl-Oxydemeton, bei dem nur das Besprühen leicht giftig ist. Pirimicarb ist absolut ungiftig.

- Wenn das Besprühen bei 21,5 "C anstatt bei 9,5 "C durchgeführt wird, sind alle Mittel wirksamer, wobei Methyl-Oxydemeton die Ausnahme bildet (Tab. 5). Bei Temperaturen über $22-23{ }^{\circ} \mathrm{C}$ und bei einer Konzentration von $3 \mathrm{~g} / \mathrm{l}$ ist Trichlorfon als Sprühmittel gefährlich, wohingegen es sich bei einer Konzentration von $1,5 \mathrm{~g} / 1$ als ungefährlich erweist, einerlei wie hoch die Temperatur ist.

- In Tabelle 6 ist der Einfluss der Dosierung für die sechs Mittel dargestellt.

- Bei Dialifor und Endosulfan jst die Wirkung der Rückstände am nachhaltigsten. Trichlorfon ist die am wenigsten beständige Substanz (deutlicher Verlust der Giftigkeit nach fünf Tagen). Phosalon liegt mit seiner Wirkung dazwischen.

\section{Diskussion und Schlussfolgerung}

Tabelle 8 gibt eine Aufstellung der Toxizität der untersuchten Insektizide in Laborversuchen. Eine Verallgemeinerung auf natürliche Bedingungen wäre unangebracht. Man kann jedoch schon jetzt feststellen, dass zwei dieser Erzeugnisse gefährlich sind : Dialifor und Endosulfan, zwei sind harmlos oder doch weniger gefährlich : Pirimicarb und Methyl-Oxydemeton. Phosalon und Trichlorfon erweisen sich bei Anwendung nach Gebrauchsanweisung als ziemlich gefährlich. Bei dem zuletzt genannten Mittel gab es widersprüchliche Ansichten zu denen der Amerikaner, was dazu führte, die Mittel bei Einbruch der Dunkelheit anzuwenden.

Unsere Ergebnisse zeigen, dass Pflanzenschutzmittel mit der Bezeichnung « nicht giftigr für Bienen m manchmal tatsächlich sehr giftig für solitäre Arten, wie Megachile pacifica sind (z. B. Endosulfan). Es kann aber auch das Gegenteil der Fall sein (Beispiel : Methyl-Oxydemeton). In der Gebrauchsanweisung der Pflanzenschutzmittel sollte daher der Hinweis " nicht giftig für Bienen » ersetzt werden durch “ nicht giftig für die Honigbiene (Apis mellifica) 》.

\section{RÉFÉRENCES BIBLIOGRAPHIQUES}

Cairaschi E. A. - Communication personnelle.

Johansen C. A., 1969. - The bee poisoning hazard from pesticides. Wash. Agr. Exp. Sta. Bull., 709, 8 p.

Johansen C. A., 1971. - How to reduce poisoning of bees from pesticides. Coop. Ext. Serv. Wash. Sta. Univ. E. M., 3473,6P.

Johansen C. A., Jaycox K. R., Hutr R., 1963. - The effect of pesticides on the alfalfa leafcutting bee. Wash. Agr. Exp. Sta., Circ., 418, 12 p. 
Johansen C. A., Eves J. D., 1967. - Toxicity of insecticides to the Alkali bee and the alfalfa leafcutting bee. Wash. Agr. Exp. Sta. Circ., 475, 15 p.

Joahnsen C. A., Eves J. D., 1972. - Acidified sprays, pollinator safety and integrated pest control on alfalfa grown for seed. J. Econ. Entomol., 65, 546-551.

Johansen C. A., Eves J. D., 1973. - Development of a pest management program on alfalfa grown for seed. Environ. Entomol. 2, 515-517.

Mizuta H. M., Johansen C. A., 1972. -- The hazard of plant-systemic insecticides to nectarcollecting bees. Wash. Agr. Exp. Sta., Bull. 72,8 p.

TAser J. N., 1977. - Méthode de test de toxicité des pesticides applicable aux abeilles solitaires et plus particulièrement à Megachile pacifica Panz (Hymenoptera, Megachilidae). Apidologie, 1977, 8 .

Torchio P. F. - Communication personnelle.

Torciro P. F., 1973. - Relative toxicity of insecticides to the honey bee, Alkali bee and alfalfa leafcutting bee (Hymenoptera Apidae, Halictidae, Megachilidae). J. Kansas Fntomol. Soc., 46, 446-453. 